\title{
Real-time membrane fusion of giant polymer vesicles
}

Yongfeng Zhou and Deyue Yan*

\section{College of Chemistry \& Chemical Engineering, State Key Laboratory of Metal Matrix Composites, Shanghai Jiao Tong University, 800 Dongchuan Road, Shanghai 200240, P. R. China}

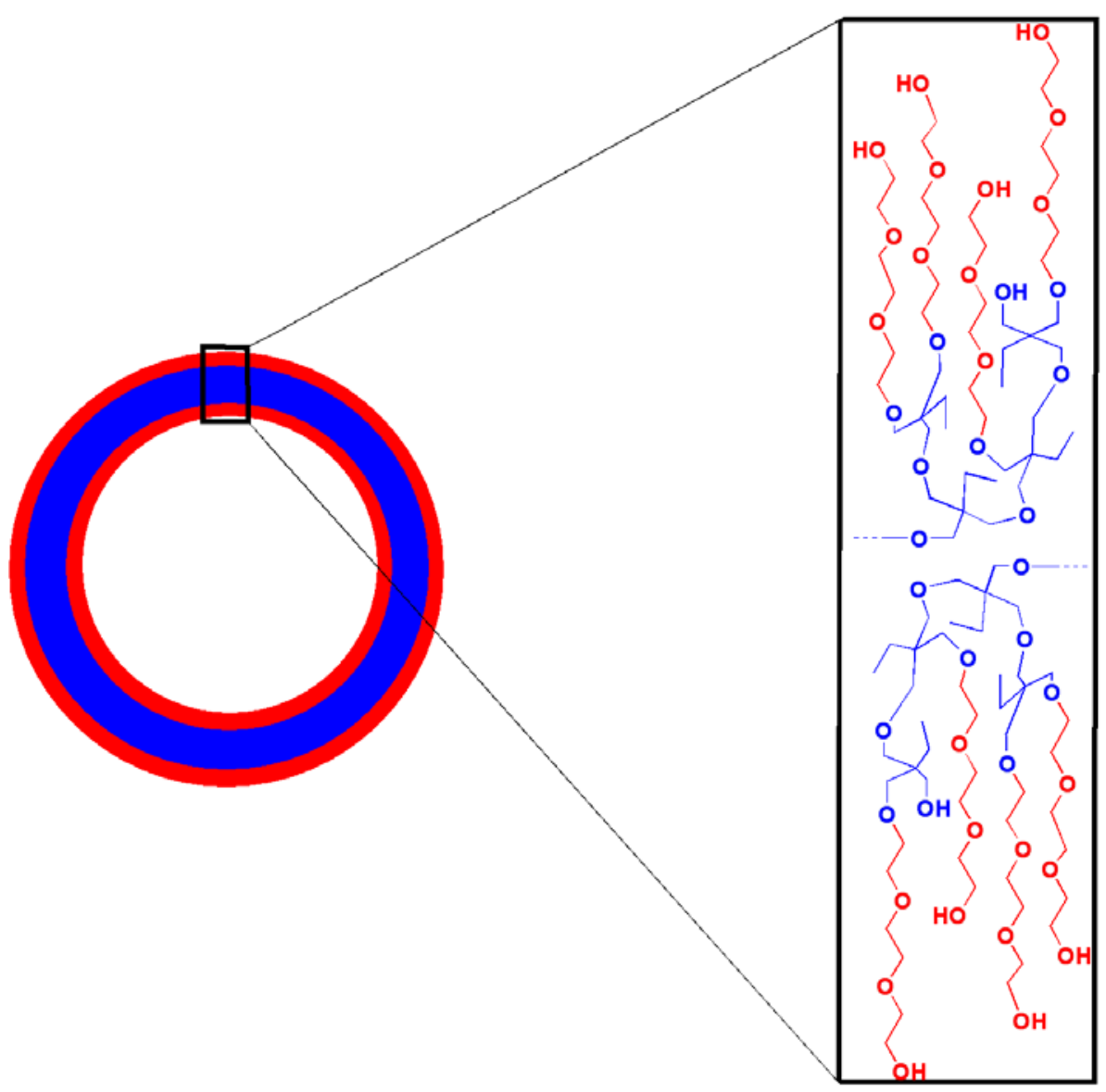

Figure S1 Proposed molecular packing model of HB1 vesicles. Left: the view of the HB1 vesicle that possesses a sandwich-like structure with an internal layer of HBPO cores and outer shells of PEO arms. The blue zone represents the condensed HBPO cores; and the red zones denote PEO arms. Right: the view of a locally magnified bilayer structure of the vesicle wall. The HBPO core is in blue and the PEO arms are in red. The illustration is not drawn to scale. HB1 vesicles were formed by the molecular self-assembly of an amphiphilic multi-arm copolymer of HBPO-star-PEO having a hydrophobic hyperbranched poly(3-ethyl-3-oxetanemethanol) (HBPO) core and a large population of hydrophilic polyethylene oxide (PEO) arms. The molecular self-assembly process of HB1 vesicles involved directly putting the polymer into the deionized water (polymer concentration $10 \mathrm{mg} / \mathrm{ml}$ ) under stirring condition at room temperature. The HB1 copolymer has a HBPO core with the molecular weight of $6,400 \mathrm{~g} / \mathrm{mol}$. The number-average molecular weight and the hydrophilic PEO volume fraction of HB1 copolymers are 12,200 and 0.69 , respectively. 


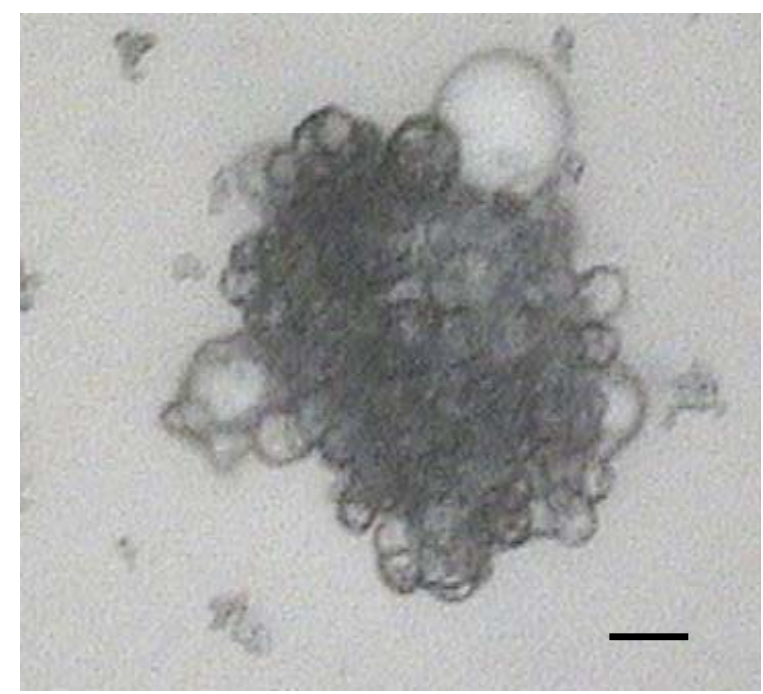

Figure S2 The aggregation of HB1 vesicles in aqueous solution. Some HB1 vesicles in aqueous solution aggregated together to form a structure like a cluster of grapes. The scale bar represents $50 \mu \mathrm{m}$. The similar morphologies of aggregated lipid vesicles have also been observed. ${ }^{1}$ 


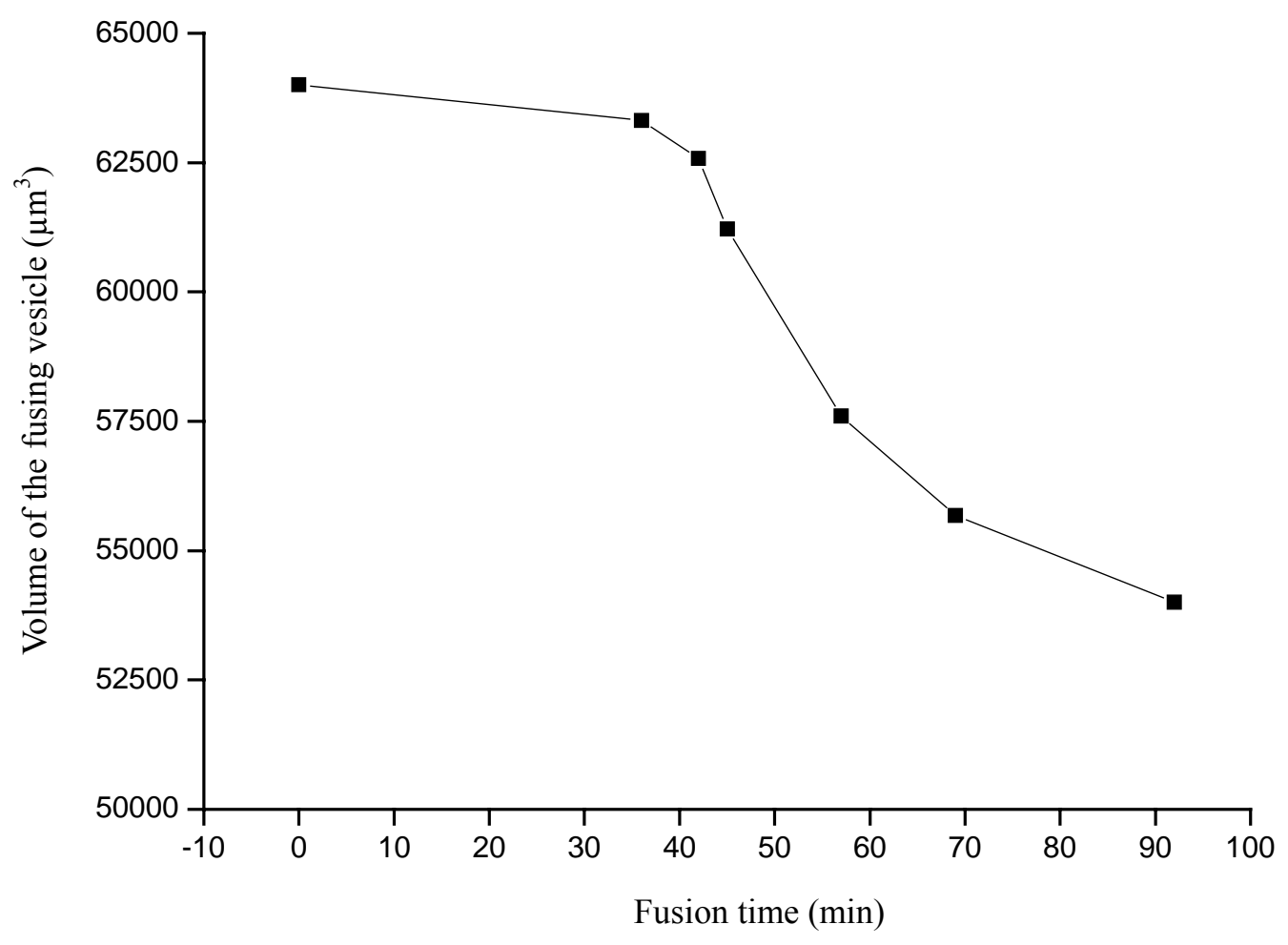

Figure S3 Variation of the volume of fusing vesicles in Figure 1. The fusing vesicles with the shape of " 8 " shape, peanut (pear) shape or oblate sphere were looked as the combination of the circular truncated cone and spherical cap during the volume calculation. 

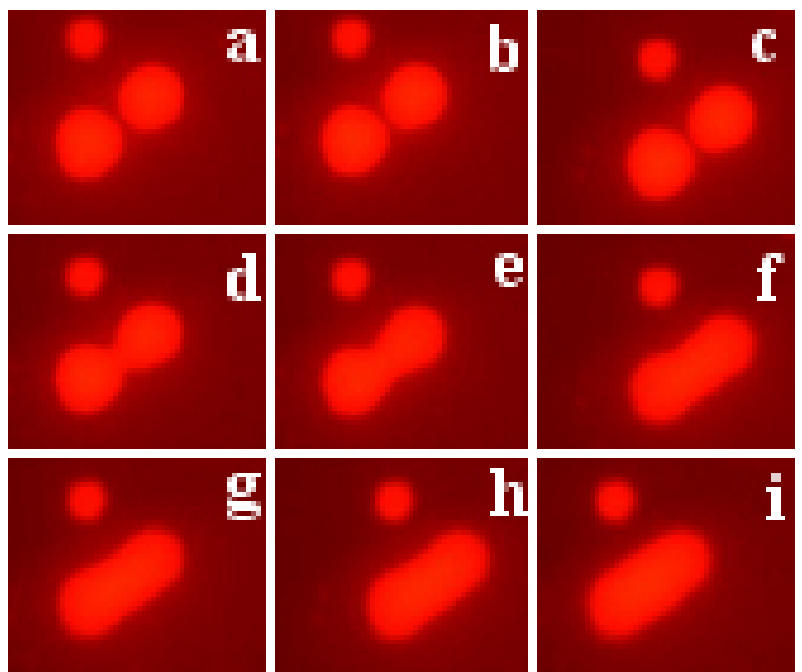

Figure S4 The real-time fusion images of two giant polymer vesicles encapsulated with hydrophilic rhodamine B. For the experiment, HB1 copolymers were directly put into the rhodamine B aqueous solution $\left(8 \times 10^{-4}\right.$ $\mathrm{mol} / \mathrm{l}$ ) with the polymer concentration of $10 \mathrm{mg} / \mathrm{ml}$, and then the solution was pretreated by ultrasound for 5 minutes $(150 \mathrm{~W}, 25 \mathrm{KHz})$. The sonicated vesicle suspension was directly observed by a fluorescence microscopy (Leica DM 4500 B) equipped with a color video recorder (leica DFC 300FX, 3.15 million pels) and a 100-watt mercury lamp at $20^{\circ} \mathrm{C}$ immediately. No attempt of purification such as the removal of free rhodamine had been tried during the experiments. The images were recorded and analyzed with the Leica Qwin plus software. The rhodamine-encapsulated HB1 vesicles are red under the fluorescence microscopy. The red content of two vesicles mixed during fusion (a-i), which provides direct evidence to support the content mixing for HB1 vesicle fusion. 


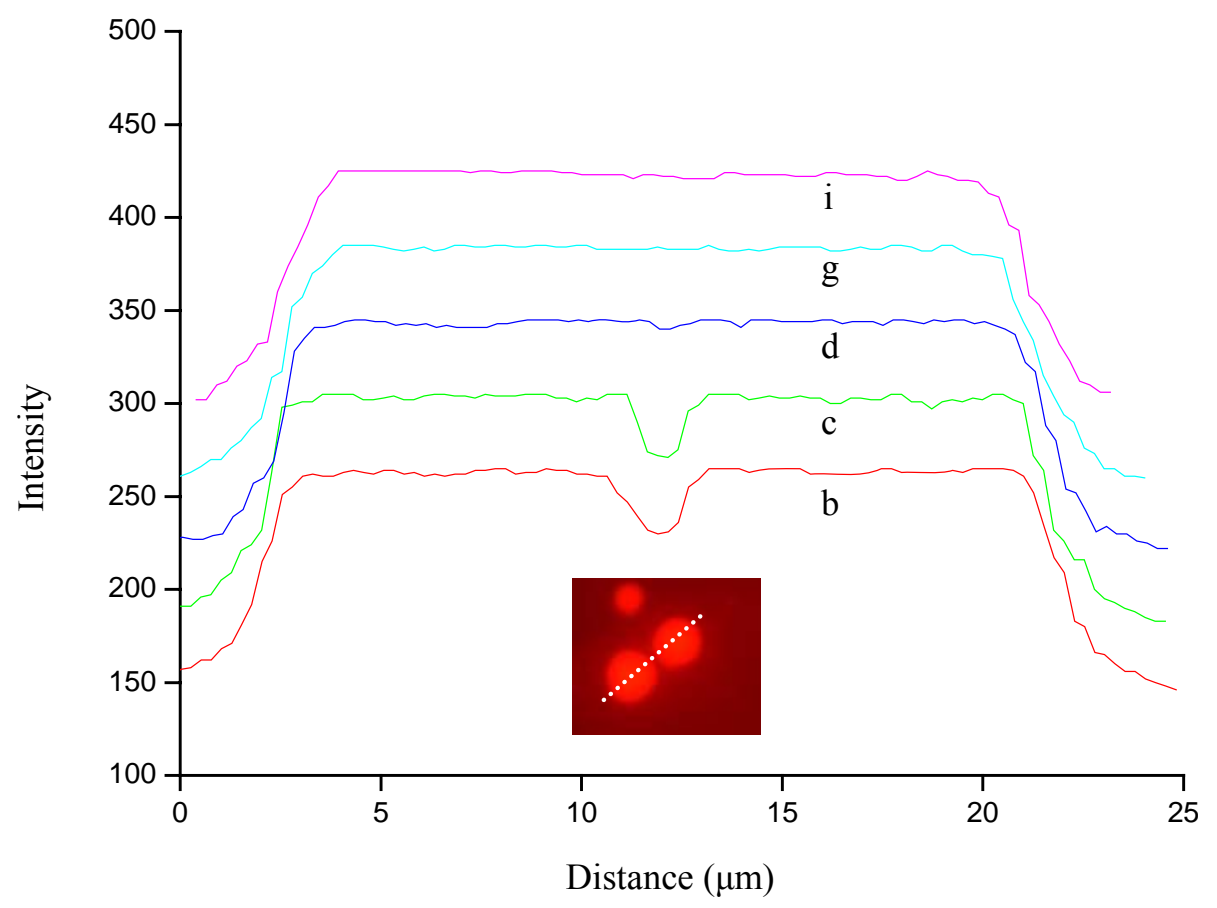

Figure S5 The spectral distribution along the axes direction of the fusing vesicle shown in Figure S4. The axes direction is shown at the bottom vesicle image with white dashed line. Before fusion (b and c), there is a wave trough that indicates the distance between the apposing vesicles, and the two platforms aside the wave trough show two separate vesicles. The wave trough becomes narrower with the vesicle apposition (b-c). Finally, the wave trough disappears completely and the original two platforms merge into one platform after membrane fusion $(\mathrm{d}, \mathrm{g}, \mathrm{i})$, which indicates that during vesicle fusion the two original vesicles have merged into one vesicle and the encapsulated contents have mixed together. The platform ( $g$ and i) also indicates that the mixed contents of the fusing vesicle have uniformly distributed throughout the vesicle. 

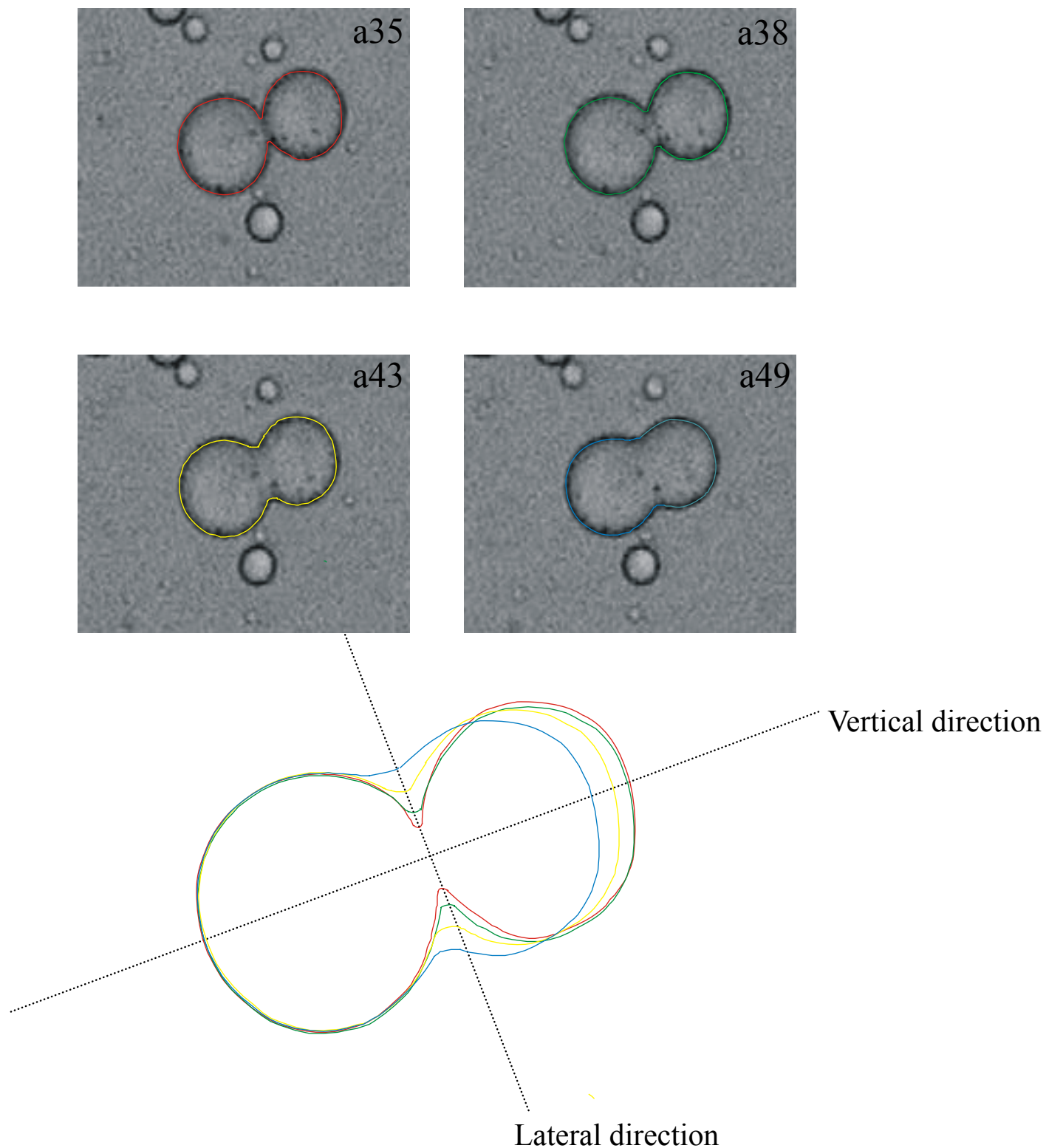

Figure S6 The images of the fusing vesicle. The optical micrographs of the vesicles are taken from Figure 1 (a35, a38, a43 and a49) in the main text. The color circles display the profiles of the fusing vesicles. The overlapped profiles at the bottom show the comparison of the fusing vesicles at various elapsed times, and the profiles (color circles) are magnified all together. Each of the color circles was drawn to coincide with the corresponding vesicle in the optical micrographs by using CorelDRAW software. The cross dash lines were drawn to show the lateral and vertical directions of the vesicles. 


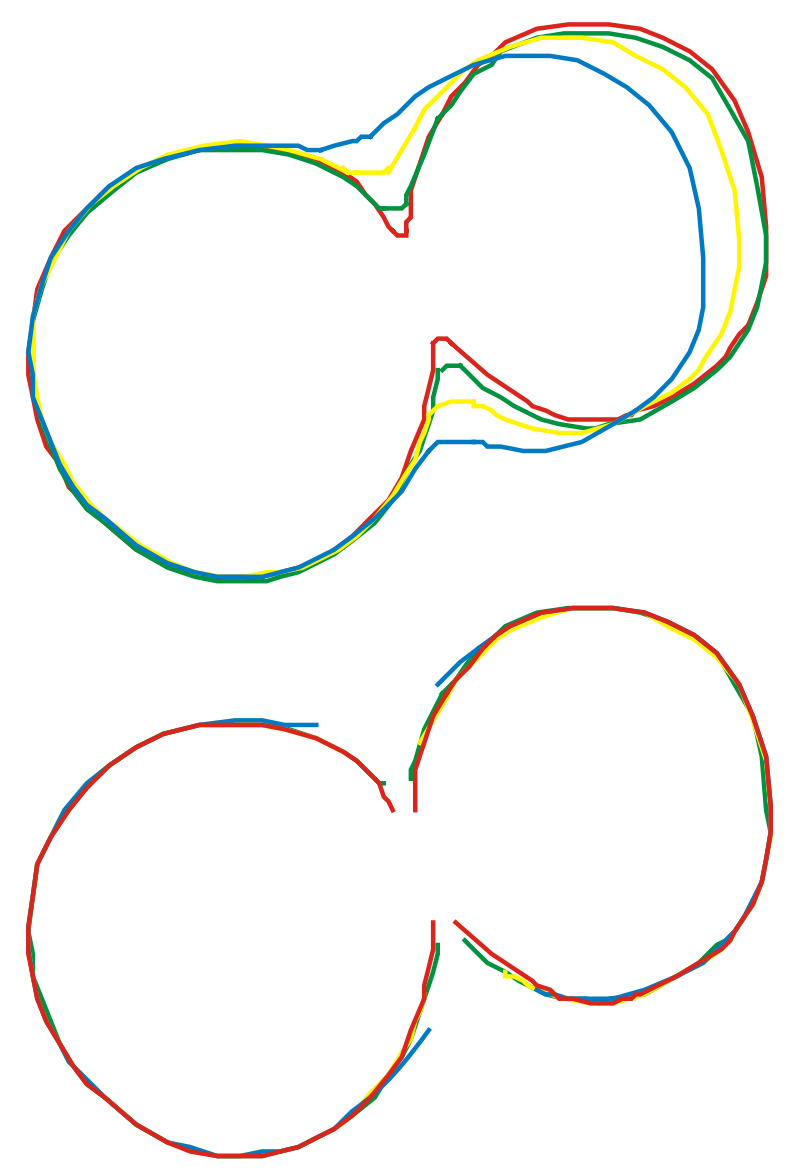

Figure S7 Comparison of the fusing vesicles in Figure 1 (a35, a38, a43 and a49) of the main text by overlapping the profiles of the vesicles at various elapsed times. The magnified color circles displaying the profiles of the fusing vesicles are the same as those in Figure S6. The drawing at the top shows the intact profiles of the fusing vesicles. The drawing at the bottom shows the overlapping of the fusing vesicles without necks. For the bottom drawing, every fusing vesicle was divided into two parts (left part and right part) by deleting the neck domain, and then the left parts and right parts of the fusing vesicles were overlapped independently. 


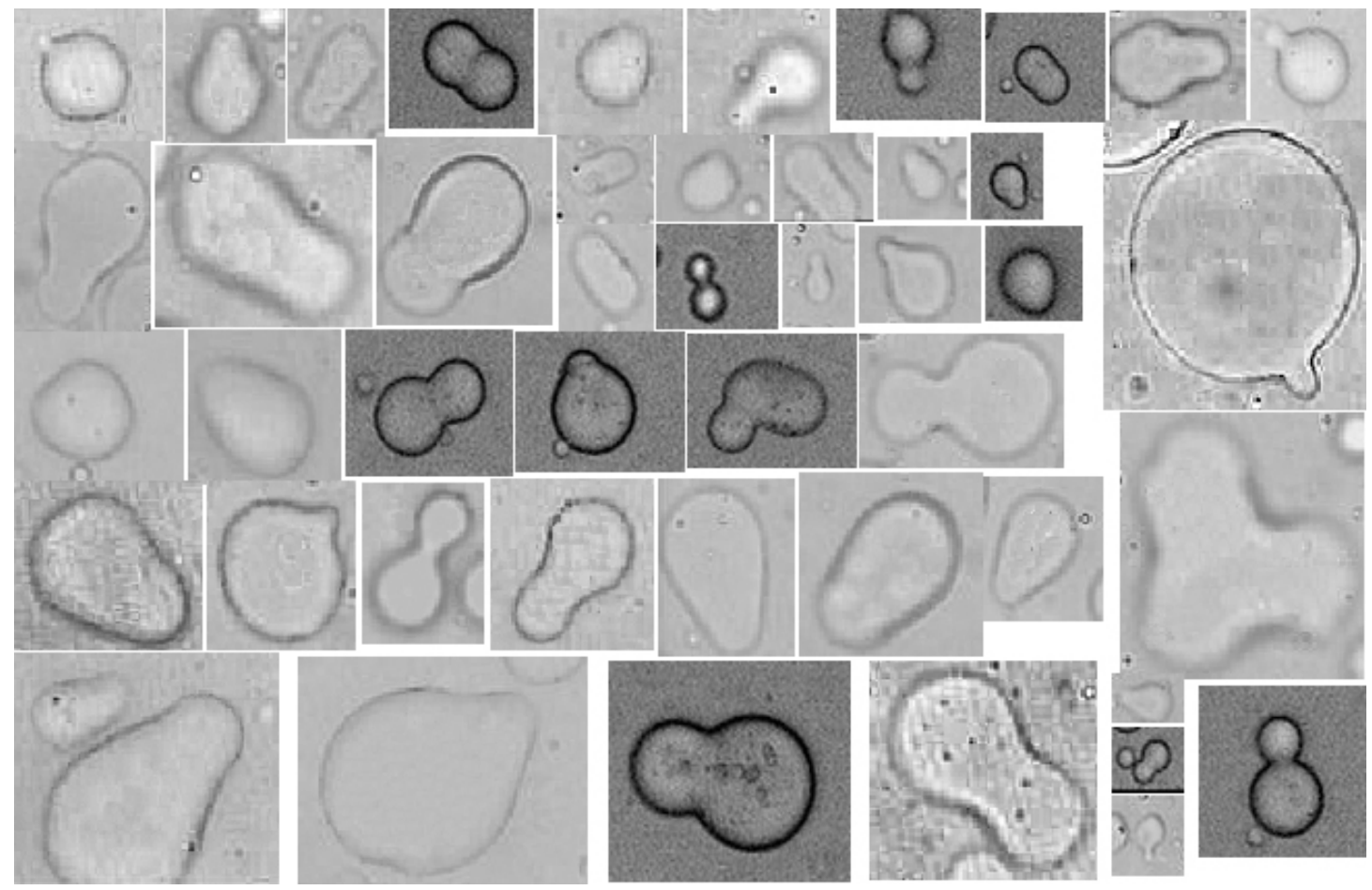

Figure S8 Part of the fusing vesicles. All the images are taken from the photos of HB1 vesicles after ultrasonic processing. These vesicle morphologies are considered as the standards to determine the happening of fusion events. A vesicle that has the similar morphology like those in the Figure is assumed in fusion.

\section{The experiments for the statistics of fusion frequency of HB1 vesicles (Figure 2 in main text):}

The HB1 vesicle aqueous solution was pretreated by ultrasound for several minutes $(150 \mathrm{~W}, 25 \mathrm{KHz})$, and then the vesicle sample was observed immediately by an optical microscopy (Leica Dmlp, TMS94) at $20{ }^{\circ} \mathrm{C}$. The morphologies of the vesicles in the field of view of the optical microscopy were recorded with the computer in synchronization. During the observation, the sample was moved at a time interval of 3 seconds. By this way, the vesicles throughout the solution were observed and recorded. The static number of the fusion frequency within $5 \mathrm{~min}$ after sonication was calculated from about one hundred micrographs of the vesicles. Every vesicle whose morphology is similar to one of those in Figure S8 is counted as a fusion event. The final static number of fusion events in Figure 2 is the average value of three round's measurements. 


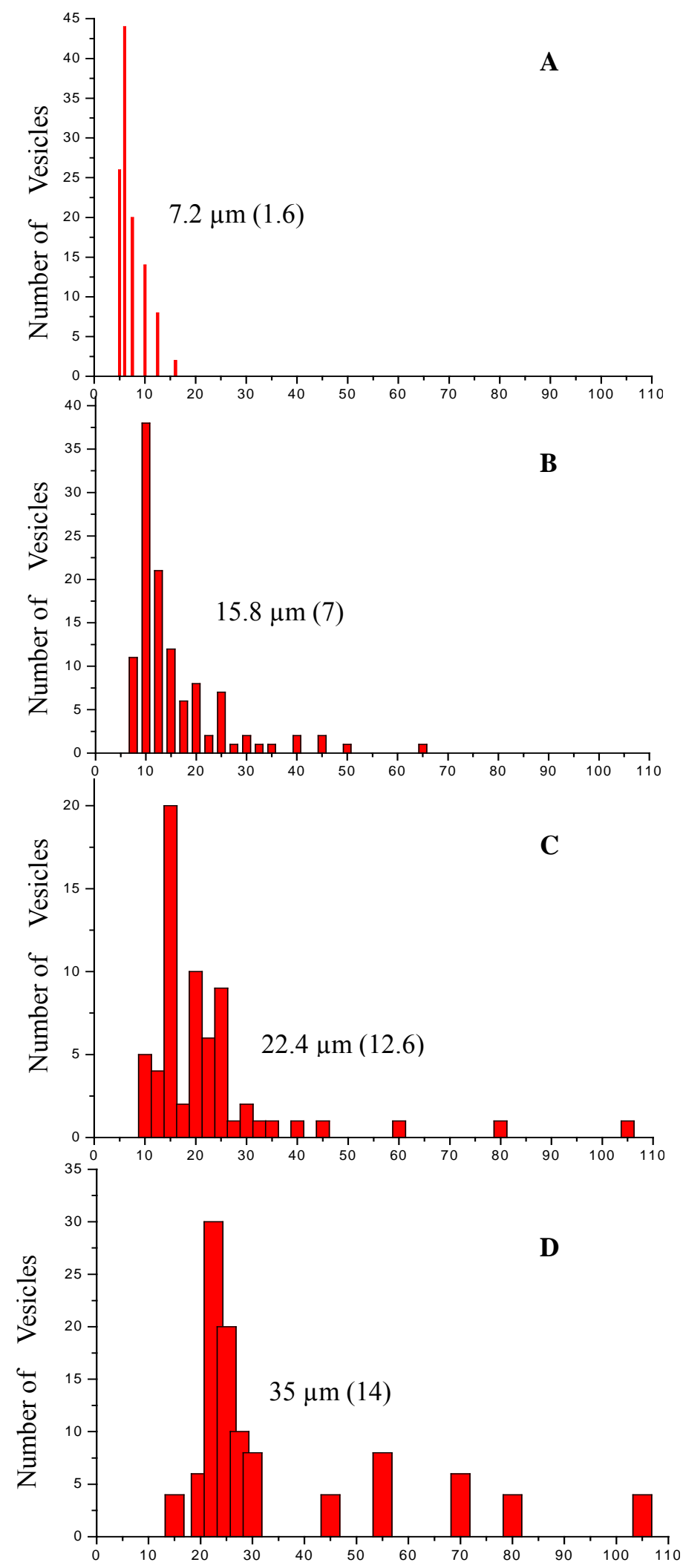

Vesicle diameter $/ \mu \mathrm{m}$

Figure S9 Variation of the size distribution of sonicated HB1 vesicles. HB1 vesicles were pretreated by ultrasound for 5 minute. The elapsed time after sonication in (A), (B), (C), (D) is 0 , $5,10,20$ minutes, respectively. The number above the bar chart in every plot shows the average diameter of the vesicles with the standard deviation (in the parentheses) for each size distribution. The size of vesicles was measured from the printed photos of the vesicles. 


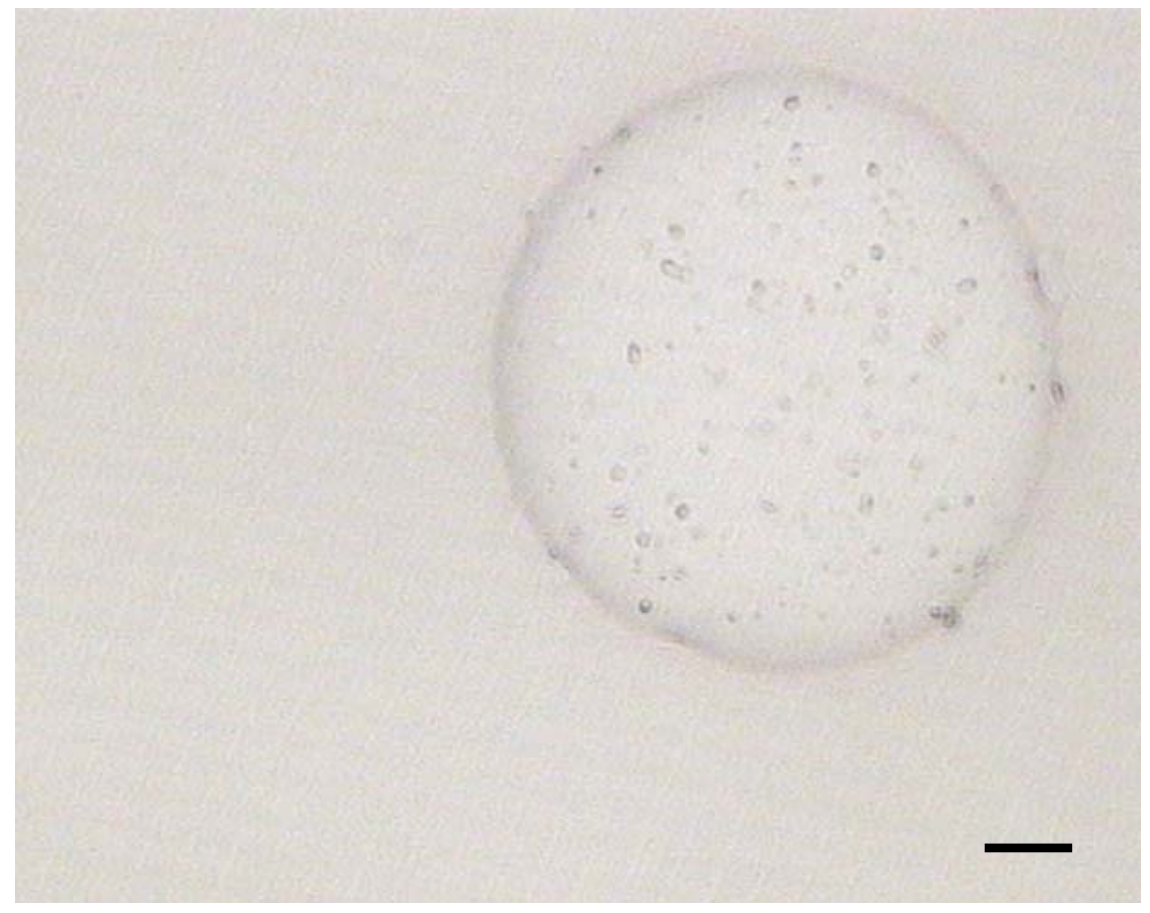

Figure S10 Typical morphology of the precipitated HB1 vesicles. The scale bar represents $25 \mu \mathrm{m}$. Some HB1 vesicles precipitated from the solution during fusion and adhered on the bottom of the culture dish, which inhibited the further fusion process. The precipitated vesicle does not have a clear rim (like a ring) under the optical microscopy, however, the rim of the vesicles suspending in the solution is much clearer. 


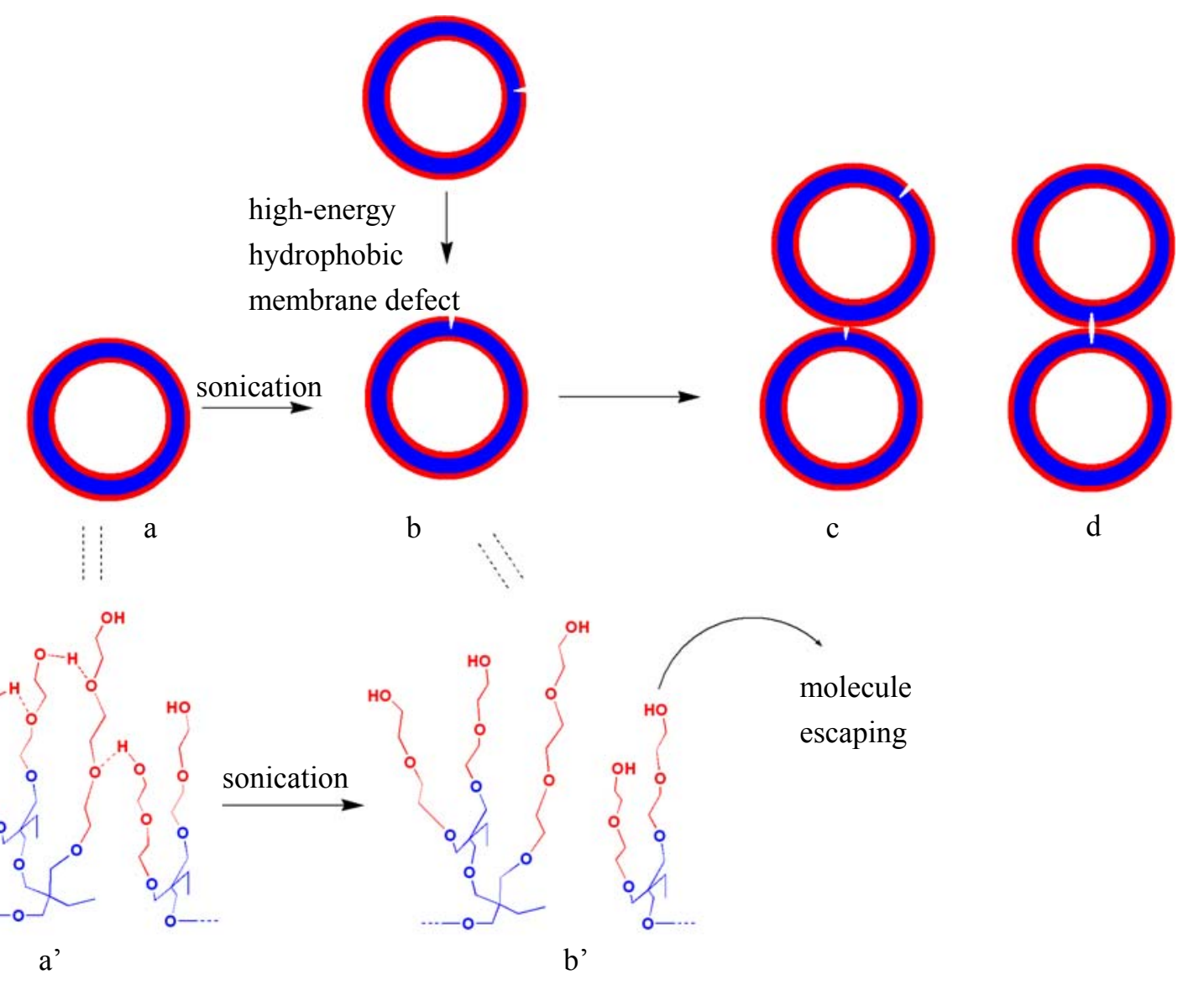

\section{Outer leaflet:}

With intermolecular and intramolecular hydrogen bonds

\section{Outer leaflet:}

Hydrogen bonds were broken by sonication

Figure S11 The proposed "defect" model of the sonicated vesicle membrane. Bottom, the view of the locally magnified outer leaflet of the HB1 bilayer vesicle (a', b'). The inner leaflet is not shown for clarity. Upper, the supposed membrane defects $(a, b)$ in the sonicated HB1 vesicles and subsequent apposition ways of vesicles(c, d). According to the previous paper reported by Lentz, the perturbation in the outer leaflet of vesicles is enough to induce vesicle fuion. ${ }^{2}$ Before sonication, the outer leaflet of HB1 vesicle has a tighter packing because of the hydrophobic interaction and the formation of inter-(intra)molecular hydrogen bonds (a, a'). ${ }^{3}$ After sonication, the local molecular packing of several domains in the outer leaflet of the vesicles becomes incompact and probably some molecules will escape from the vesicle wall into the bulk aqueous solution because sonication may partially break the hydrogen bonds and destabilize the membrane (b'), which leads to the "high-energy hydrophobic membrane defect" ${ }^{4}$ due to the exposure of the hydrophobic lamella of the vesicle into water (b). ${ }^{2}$ The defects in (b) are similar to those of sonicated liposomes as suggested by Cevc and coworker. ${ }^{5}$ There are two possible apposition styles for the sonicated vesicles with defects (c and d). To heal the hydrophobic defect, the molecules from the outer monolayer of the apposed target membrane are probably recruited to merge into the defect zone, which is believed to be a trigger for the membrane fusion theoretically. ${ }^{4,6}$ 


\section{Reference:}

(1) (a) Menger, F. M.; Balachander, N. J. Am. Chem. Soc. 1992, 114, 5862. (b) Bergstrand, N.; Arfvidsson, M. C.; Kim, J.-M.; Thompsonb, D. H.; Edwardsa, K. Biophys. Chem.2003, 104, 361. (c) Boomer, J. A.; Inerowicz, H. D.; Zhang, Z.; Bergstrand, N.; Edwardsa, K.; Kim, J.-M.; Thompson, D. H. Langmuir 2003, 19, 6408. (d) Teschke, O.; Souza, E. F. Langmuir 2002, 18, 6513. (e) Bizzozero, O. A.; Howard, T. A. Neurochem. Res., 2002, 27, 1269. (f) Zschörnig, O.; Richter, W.; Paasche, G.; Arnold, K. Colloid Polym. Sci. 2000, 278, 637.

(2) Lee, J.; Lentz, B. R. Biochemistry 1997, 36, 421.

(3) Zhou, Y.; Yan, D. Angew. Chem. Int. Ed. 2004, 43, 4896.

(4) Bentz, J. Biophys. J. 2000, 78, 886.

(5) Cevc, G.; Richardsen, H. Adv. Drug Deliv. Rev. 1999, 38, 207.

(6) Marrink, S. J.; Mark, A. E. J. Am. Chem. Soc. 2003, 125, 11144. 